Research Paper

\title{
ERCC1-expressing circulating tumor cells as a potential diagnostic tool for monitoring response to platinum-based chemotherapy and for predicting post-therapeutic outcome of ovarian cancer
}

\author{
Issam Chebouti ${ }^{1,6}$, Jan Dominik Kuhlmannn ${ }^{2,5,6}$, Paul Buderath ${ }^{1,6}$, Stephan Weber ${ }^{3}$, \\ Pauline Wimberger ${ }^{2,5,6}$, Yvonne Bokeloh4, Siegfried Hauch ${ }^{4}$, Rainer Kimmig ${ }^{1,6}$, \\ Sabine Kasimir-Bauer ${ }^{1,6}$ \\ ${ }^{1}$ Department of Gynecology and Obstetrics, University Hospital Essen, Essen, Germany \\ ${ }^{2}$ Department of Gynecology and Obstetrics, Medical Faculty and University Hospital Carl Gustav Carus, Technische Universität \\ Dresden, Dresden, Germany \\ ${ }^{3}$ ACOMED Statistik, Department of Biostatics, Magdeburg, Germany \\ ${ }^{4}$ QIAGEN Hannover GmbH, Langenhagen, Germany \\ ${ }^{5}$ National Center for Tumor Diseases (NCT), Partner Site Dresden, Dresden, Germany \\ ${ }^{6}$ German Cancer Consortium (DKTK), Dresden and German Cancer Research Center (DKFZ), Heidelberg, Germany \\ Correspondence to: Issam Chebouti, email: Issam.Chebouti@uk-essen.de \\ Keywords: circulating tumor cells, ERCC1, platinum-resistance, ovarian cancer, prognosis \\ Received: June 30, $2016 \quad$ Accepted: October 13, $2016 \quad$ Published: November 11, 2016 \\ Copyright: Chebouti et al. This is an open-access article distributed under the terms of the Creative Commons Attribution License \\ (CC-BY), which permits unrestricted use, distribution, and reproduction in any medium, provided the original author and source \\ are credited.
}

\section{ABSTRACT}

Background: We recently showed that the presence of ERCC1+CTCs is an independent predictive biomarker for platinum-resistance and poor prognosis of ovarian cancer. The goal of our current research was to determine how the auxiliary assessment of ERCC1-transcripts influences overall CTC-detection rate. We extended this investigation from an initially predictive setting to paired pre- and posttherapeutic blood analysis in order to see, whether ERCC1+CTCs dynamics mirror response to chemotherapy.

Methods: 65 Paired blood samples $(10 \mathrm{ml})$ of primary ovarian cancer patients at primary diagnosis and after chemotherapy were studied for CTCs with the AdnaTest Ovarian Cancer (QIAGEN Hannover $\mathbf{G m b H}$ ). We analyzed the tumor-associated transcripts EpCAM, MUC-1 and CA-125. ERCC1-transcripts were investigated in a separate approach by singleplex RT-PCR.

Results: Auxiliary assessment of ERCC1-transcripts enhanced the overall CTCdetection rate up to $17 \%$. ERCC1 ${ }^{+}$CTCs (defined as positive for one of the AdnaTest markers plus ERCC1-positivity) were detected in $15 \%$ of patients at primary diagnosis and in $12 \%$ after chemotherapy. The presence of ERCC1 ${ }^{+}$CTCs after chemotherapy correlated with platinum-resistance $(P=0.01)$, reduced $P F S(P=0.0293)$ and $0 S$ $(P=0.0008)$ and their persistence indicated poor post-therapeutic outcome (PFS: $P=0.005$; OS: $P=0.0058$ ). Interestingly, the assessment of ERCC1-transcripts alone was sufficient for the detection of prognostic relevant ERCC1-expressing CTCs.

Conclusion: Auxiliary assessment of ERCC1-transcripts expands the phenotypic spectrum of CTC detection and defines an additional overlapping fraction of ERCC1expressing CTCs, which are potentially selected by platinum-based chemotherapy. Specifically, we suggest that ERCC1+CTCs could additionally be useful as a surrogate for monitoring platinum-based chemotherapy and to assess the post-therapeutic outcome of ovarian cancer. 


\section{INTRODUCTION}

Epithelial ovarian cancer is the fifth leading cause of cancer death of women in Europe and the United States and the second most common gynecological malignancy [1]. Most cases are diagnosed in advanced stages and, although response rates to chemotherapy reach up to $80 \%$, the majority of patients cannot be cured. Standard treatment of advanced ovarian cancer is primary surgery aiming at complete macroscopic tumor resection followed by platinum- and paclitaxel-based chemotherapy, which has been shown to prolong progression free survival (PFS) as well as overall survival (OS) [2]. Postoperative residual tumor is one of the most important prognostic factors in advanced ovarian cancer [3-5]. However, despite advances in treatment, more than half of all patients will experience recurrence, resulting in poor overall survival [6].

Importantly, resistance to platinum-based chemotherapy, which can be caused by e.g. enhanced DNA-repair capacity of tumor cells, occurs in about 15$20 \%$ of patients and constitutes one of the most recognized clinical challenges for ovarian cancer [7]. The nucleotide excision repair (NER) pathway is a key pathway involved in mediating resistance or sensitivity to platinum-based chemotherapeutic agents. The excision repair crosscomplementation group 1 (ERCC1) protein plays a key role in NER. It dimerizes with xeroderma pigmentosum complementation group $\mathrm{F}$ (known as ERCC4) and mediates the excision of DNA-platinum adducts, typically induced by platinum-based chemotherapy [8]. ERCC1expression has been extensively studied in primary tumor tissue of several cancer entities, including ovarian cancer, and has been proposed as a potential predictor for response to platinum-based chemotherapy. However, this concept has been controversial, particularly in the context of immunohistochemical ERCC1-detection, and has not been implemented into clinical routine so far [9-20]. Taking into account that primary tumor tissue is typically available only at primary diagnosis, it would be valuable to establish a non-invasive blood-based biomarker for stratifying response to platinum-based chemotherapy at primary diagnosis and for guiding individualized therapy decisions in the future. We recently showed that the presence of ERCC $1^{+} \mathrm{CTCs}$ (circulating tumor cells) at primary diagnosis of ovarian cancer, a potentially platinum-resistant CTC-subgroup, is an independent predictive biomarker for primary platinum-resistance and poor prognosis of ovarian cancer [21].

We now explored in more detail, in how far the auxiliary assessment of ERCC1-transcripts influences overall CTC-detection rate and whether this molecular marker may improve the phenotypic range of CTCdetection by the AdnaTest Ovarian Cancer platform. We essentially extended this investigation from an initially predictive setting to paired pre- and post-therapeutic blood analysis and explored clinical relevance of ERCC ${ }^{+} \mathrm{CTC}$ dynamics in response to platinum-based chemotherapy.

\section{RESULTS}

\section{Influence of auxiliary ERCC1-transcript assessment on the $\mathrm{CTC}$-detection rate}

We previously have demonstrated that ERCC1 extends clinical information of CTCs as a prognostic biomarker to the prediction of platinum-resistance at primary diagnosis of ovarian cancer [21]. We now explored in more detail how additional assessment of ERCC1 influences the overall detection rate of CTCs in 65 paired pre-operative and post-chemotherapeutic blood samples from ovarian cancer patients. First we assessed the marker transcripts according to the AdnaTest Ovarian Cancer in its previous configuration. CTC-positivity of this assay was indicated by the detection of at least one of the transcripts EpCAM, MUC-1 or CA-125, herein referred to as "AdnaTest ${ }^{\text {"'. }}$. Furthermore, we now considered ERCC1-transcripts as an additional marker for CTC-detection.

Figure 1A summarizes the detected CTC-types and shows their relative proportions among the studied ovarian cancer patients. In $8 \%$ of patients AdnaTestpositivity was exclusively observed. In $17 \%$ we detected exclusively ERCC1-positive CTCs and in 15\% we observed dual positivity for the AdnaTest and ERCC1. Subsequently, we were interested in how auxiliary assessment of ERCC1 influences the overall detection rate of CTCs in ovarian cancer. Therefore, we compared overall CTC-detection rates across several defining criteria for "CTC-positivity", with the presence of ERCC1-transcripts as an additional alternative criterion or as obligatory requirement, respectively (Figure 1B): We observed a CTC-detection rate of $23 \%$ before surgery, comprised of patients with only AdnaTest positivity and dual AdnaTest/ERCC1-positive patients. Detection rates were substantially increased up to $40 \%$ if ERCC1 was considered as a further alternative marker for CTC-positivity (AdnaTest ${ }^{+}$OR ERCC1 ${ }^{+}$). Since this CTC-definition now comprises a further subgroup of patients with exclusively ERCC1-expressing CTCs. This subgroup alone can be detected with an overall detection rate of $17 \%$ (AdnaTest AND ERCC1 $1^{+}$). Lastly, according to a more stringent definition of combined positivity (AdnaTest ${ }^{+}$AND ERCC $1^{+}$), overall detection decreased to $15 \%$.

After platinum-based chemotherapy the proportion of CTC-subtypes and their overall detection rates among the above mentioned CTC-definition criteria were grossly comparable with those found before therapy (Figure $1 \mathrm{C}+1 \mathrm{D})$.

\section{ERCC1 $^{+}$CTCs predict post therapeutic outcome}

The median follow up time for PFS was 37 months (range 4-120 months) resulting in $36(55 \%)$ relapses while 28 patients $(43 \%)$ presented with no relapse. After 
a median follow-up time of 45 months (range 11-117 months) for OS, 42 patients $(65 \%)$ were still alive and 23 patients $(35 \%)$ had died (Table 1$)$.

We first explored the clinical relevance of ERCC1expressing CTCs in post-therapeutic blood samples. For this purpose, in accordance with our previous publication [21], we primarily focused on the most stringent definition of ERCC1-expressing CTCs, which is based on the previous AdnaTest markers (EpCAM, MUC-1, CA-125) and encludes ERCC1-positivity as an additional obligatory requirement $\left(\mathrm{AdnaTest}^{+} \mathrm{AND} \mathrm{ERCC}^{+}\right.$). This cell population is from now on referred to as $\mathrm{ERCC} 1^{+} \mathrm{CTCs}$. The presence of post-therapeutic ERCC $1^{+} \mathrm{CTCs}$ significantly correlated with decreased PFS $(p=0.0293)$ and $\operatorname{OS}(\mathrm{p}=0.0008$, Figure $2 \mathrm{~A}+2 \mathrm{~B})$. Furthermore, the presence of ERCC $1{ }^{+} \mathrm{CTCs}$ after chemotherapy correlated with primary platinum-resistance $(p=0.01$, data not shown).

\section{ERCC1 $^{+}$CTC dynamics mirror response to platinum-based chemotherapy}

We were further interested in how the levels of ERCC $1^{+} \mathrm{CTCs}$ in our patients changed in response to platinum-based chemotherapy. A stratification of our study patients according to "ERCC $1^{+} \mathrm{CTC}$ dynamic subgroups" is presented in Figure 3. The majority of patients were negative for ERCC $1^{+} \mathrm{CTCs}$ throughout (77\%, "neg-neg") treatment. In $11 \%$ of patients we observed ERCC $1{ }^{+} \mathrm{CTCs}$ before surgery, which disappeared after platinum-based chemotherapy ("pos-neg"). Moreover, $8 \%$ of patients were initially negative and $\mathrm{ERCC} 1^{+} \mathrm{CTC}$ newly appeared after therapy ("neg-pos"). Finally, in 5\% of patients, persistent ERCC $1^{+} \mathrm{CTCs}$ were observed before surgery and after chemotherapy ("pos-pos").

Interestingly, patients with persistent positivity for $\mathrm{ERCC} 1^{+} \mathrm{CTC}$ before surgery and after
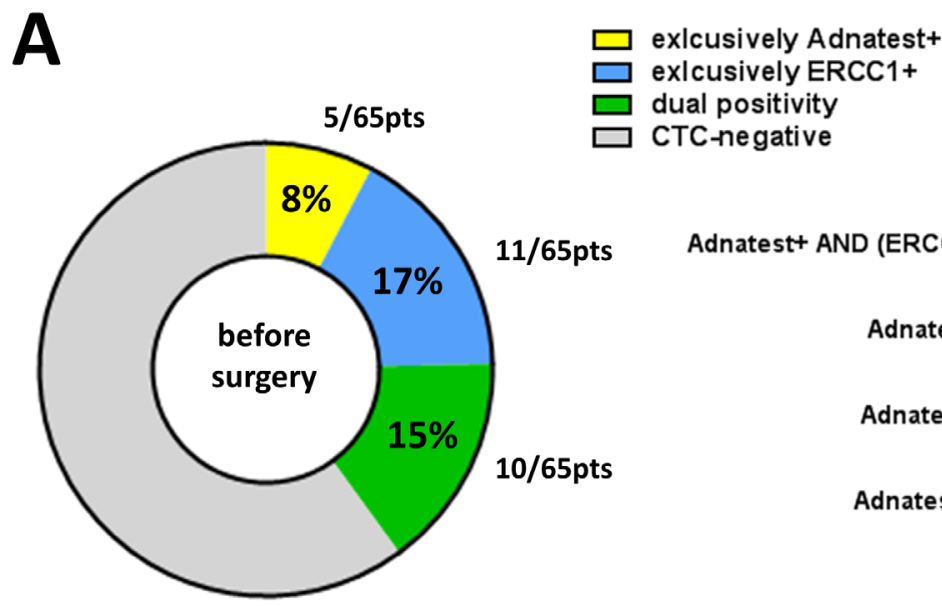

B

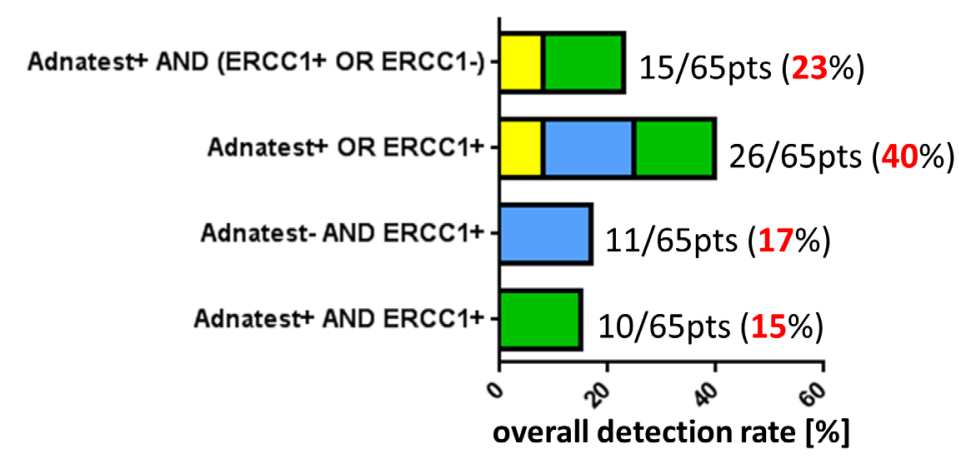

c

$5 / 65 p t s$

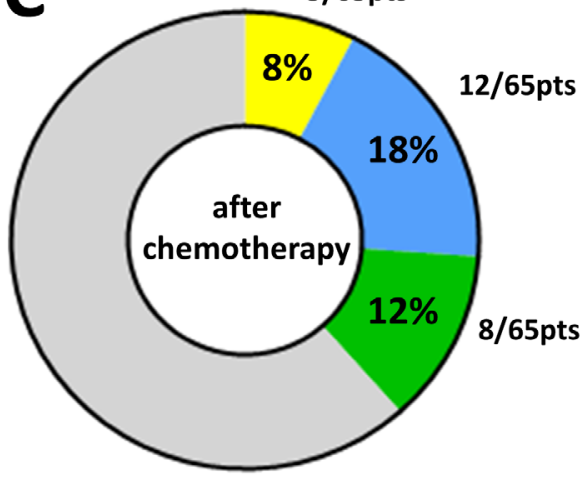

D

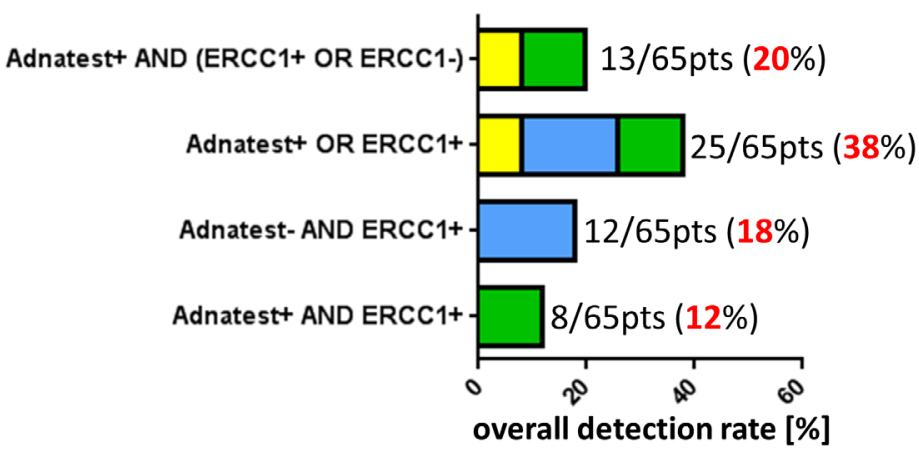

Figure 1: Influence of auxiliary ERCC1-transcript assessment on CTC-detection rate. A. The pie chart shows the different CTC-types and their relative proportions among the studied ovarian cancer patients before surgery $(\mathrm{n}=65)$. Percentages indicate the proportion of patients with exclusively-Adnatest-positivity (yellow), exclusively-ERCC1-positivity (blue), dual-positivity for Adnatest/ ERCC1 (green) and CTC-negative patients (grey). B. The stacked bar chart summarizes four CTC-definition criteria, considering ERCC1 as additional transcript marker and shows, how this is translated into different overall CTC-detection rates. C+D. These illustrations depict the same type of analysis as reported above, however refer to paired blood samples analyzed after platinum-based chemotherapy ( $\mathrm{n}=65$ ). In all figures, absolute patient numbers in each subgroup are indicated. 
Table 1: Patient characteristics at the time of primary diagnosis

\begin{tabular}{|c|c|}
\hline Total & 65 \\
\hline Age & median 61 years, $(27-92)$ \\
\hline \multicolumn{2}{|l|}{ FIGO stage } \\
\hline I-II & $11(17 \%)$ \\
\hline III & $41(63 \%)$ \\
\hline IV & $13(20 \%)$ \\
\hline \multicolumn{2}{|l|}{ Nodal status } \\
\hline $\mathrm{N}_{\mathrm{o}}$ & $24(37 \%)$ \\
\hline $\mathrm{N}_{1}$ & $28(43 \%)$ \\
\hline $\mathrm{N}_{\mathrm{x}}$ & $13(20 \%)$ \\
\hline \multicolumn{2}{|l|}{ Grading } \\
\hline I-II & $28(43 \%)$ \\
\hline III & $37(57 \%)$ \\
\hline Unknown & $0(0 \%)$ \\
\hline \multicolumn{2}{|l|}{ Residual tumor } \\
\hline \multicolumn{2}{|l|}{ Macroscopic } \\
\hline Complete resection & $38(58 \%)$ \\
\hline Any residual tumor & $27(42 \%)$ \\
\hline \multicolumn{2}{|l|}{ Histologic type } \\
\hline Serous & $52(80 \%)$ \\
\hline Mucinous & $9(14 \%)$ \\
\hline Other & $4(6 \%)$ \\
\hline \multicolumn{2}{|l|}{ Survival } \\
\hline $\mathrm{PFS}^{1}$ & median 37 months, (4-120 months) \\
\hline $\mathrm{OS}^{2}$ & median 45 months, (11-117 months) \\
\hline Alive & $42(65 \%)$ \\
\hline Dead & $23(35 \%)$ \\
\hline Unknown & $0(0 \%)$ \\
\hline \multicolumn{2}{|l|}{ Recurrence } \\
\hline No relapse & $28(43 \%)$ \\
\hline Relapse & $36(55 \%)$ \\
\hline Unknown & $1(2 \%)$ \\
\hline
\end{tabular}

${ }^{1}$ PFS: progression-free survival, ${ }^{2} \mathrm{OS}$ : overall survival

chemotherapy (ERCC1 ${ }^{+} \mathrm{CTC}$ "pos-pos") had a very poor PFS $(p=0.0053)$ and $\mathrm{OS}(\mathrm{p}=0.0058$, Figure $4 \mathrm{~A}+4 \mathrm{~B})$ compared to all other dynamic subgroups together ("neg-neg" or "pos-neg" and "neg-pos"). Furthermore, we observed the trend that patients with newly acquired
ERCC $1^{+} \mathrm{CTCs}$ after chemotherapy ("neg-pos") had a shorter PFS by trend $(p=0.2871)$ and a significantly shorter OS ( $\mathrm{p}=0.0202)$ than the "neg-neg" group and the "pos-neg" group together (Figure 4C+4D). 
The assessment of ERCC1-transcripts alone is a surrogate for the detection of prognostically relevant CTCs

We were interested in how prognostic information as described above was retained when ERCC1-transcripts alone were assessed. Therefore, we exclusively focused on ERCC1-transcript expression, irrespectively of EpCAM, MUC-1 or CA-125 positivity and re-performed survival analysis. The presence of post-therapeutic ERCC1-transcript positivity alone indicated reduced PFS $(p=0.0158)$ and $\mathrm{OS}(\mathrm{p}=0.0377$, Figure $5 \mathrm{~A}+5 \mathrm{~B})$. Once
A

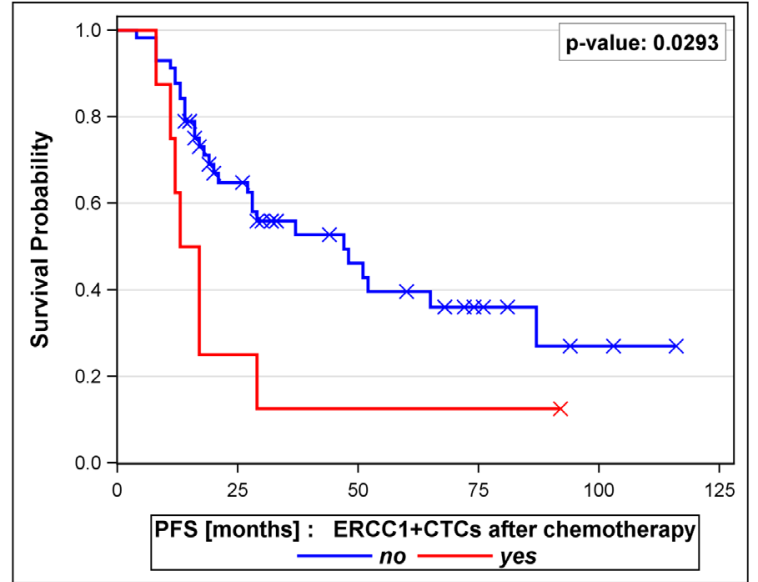

B

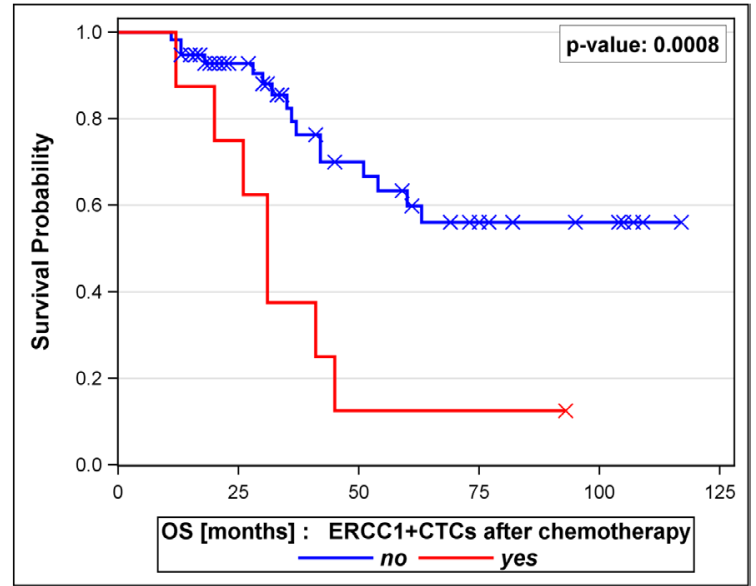

Figure 2: Prognostic relevance of ERCC1 ${ }^{+} \mathbf{C T C s}$ after chemotherapy. A patient was considered positive for $\mathrm{ERCC1} 1^{+} \mathrm{CTCs}$ if at least one of the AdnaTest transcript markers (EpCAM, MUC-1 or CA-125) was detected, in addition to ERCC1-positivity. The KaplanMeier analysis shows A. progression-free survival and B. overall survival of patients with detectable ERCC1 ${ }^{+} \mathrm{CTCs}$ after platinum-based chemotherapy (bottom curves) in comparison to patients with non-detectable $\mathrm{ERCC1} 1^{+} \mathrm{CTCs}$ (top curves).

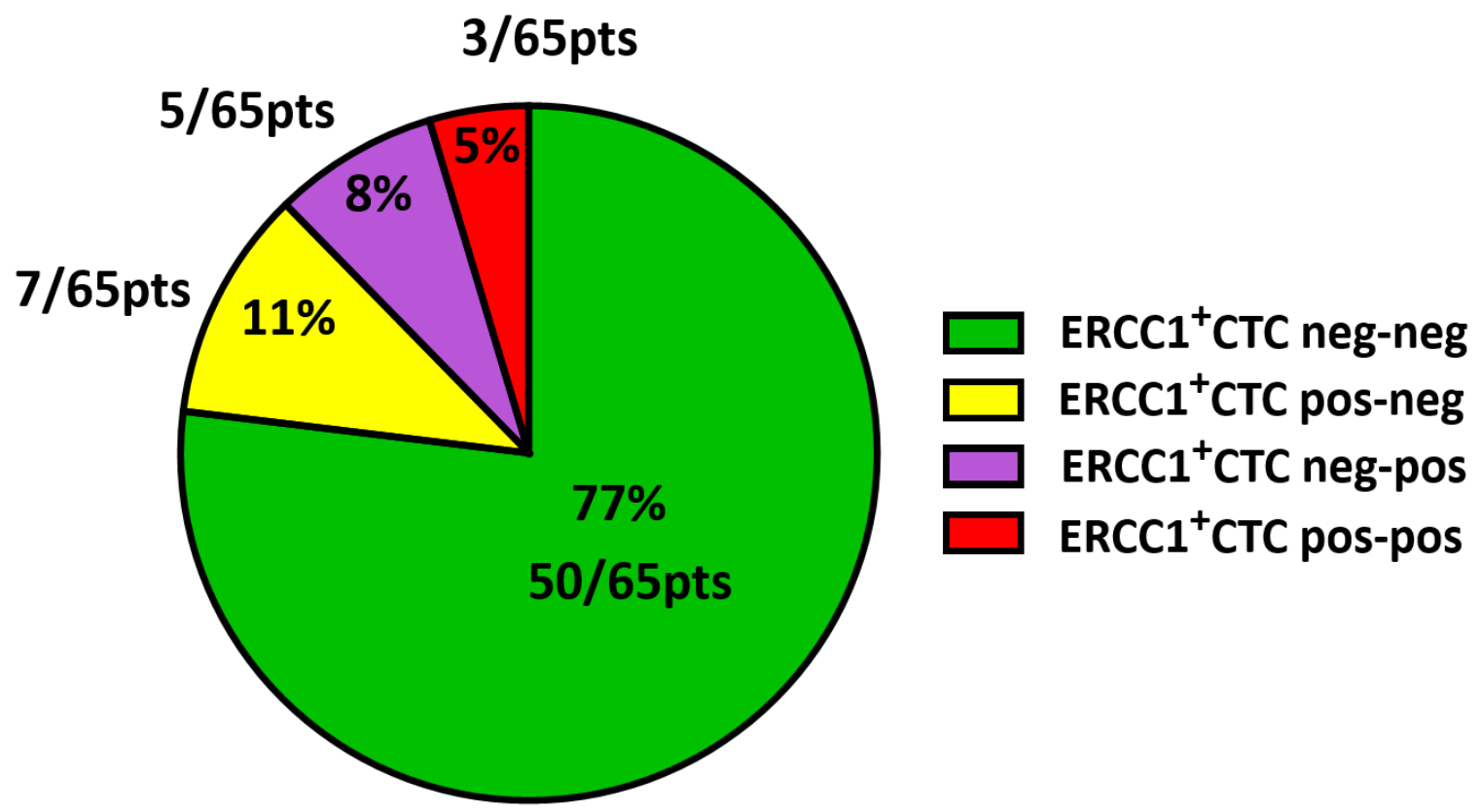

Figure 3: Dynamics of ERCC1 ${ }^{+}$CTCs in the course of platinum-based chemotherapy. A patient was considered positive for ERCC $1^{+} \mathrm{CTCs}$ if at least one of the Adnatest transcript markers (EpCAM, MUC-1 or CA-125) was detected, in addition to ERCC1-positivity. The pie chart shows a stratification of the study cohort $(\mathrm{n}=65)$ into different subgroups, according to the dynamics of $\mathrm{ERCC}{ }^{+} \mathrm{CTCs}$ before surgery and after chemotherapy. Besides the group of patients, who were negative for $\mathrm{ERCC1} 1^{+} \mathrm{CTCs}$ throughout $\left(\mathrm{ERCC1} 1^{+} \mathrm{CTCs}\right.$ neg-neg), we observed patients, who became negative after chemotherapy (ERCC1 ${ }^{+} \mathrm{CTCs}$ pos-neg), patients with newly acquired positivity after chemotherapy (ERCC1 ${ }^{+} \mathrm{CTCs}$ neg-pos) or persistently positive patients (ERCC1 ${ }^{+} \mathrm{CTCs}$ pos-pos). Percentages and absolute patient numbers are indicated. 
more, a stratification of our study patients according to "ERCC1 dynamic subgroups" was performed and is presented in Supplementary Figure S1. The majority of patients were negative for ERCC1-transcritps throughout treatment (57\%, "neg-neg"). In $12 \%$ of patients we observed ERCC1-positivity before surgery which disappeared after platinum-based chemotherapy ("posneg"). Moreover, $11 \%$ of patients were initially negative and ERCC $1^{+} \mathrm{CTCs}$ newly appeared after therapy ("negpos"). Finally, in $20 \%$ of patients, persistent ERCC $1^{+} \mathrm{CTCs}$ were observed before surgery and after chemotherapy ("pos-pos"). More interestingly, as already reported for ERCC $1{ }^{+} \mathrm{CTCs}$, the ERCC1 "pos-pos" subgroup also had a significantly decreased PFS $(p=0.0021)$ and OS $(p=0.0327)$, compared to all other dynamic subgroups together (Figure $5 \mathrm{C}+5 \mathrm{D}$ ). Of note is that the statistical significance level of these findings was generally lower compared to the prognostic relevance of ERCC $1{ }^{+} \mathrm{CTCs}$, which referred to combined CTC- and ERCC1-positivity.

The number of patients at risk in each of the subgroups shown in Figures 2, 4 and 5 are documented in Supplementary Table S1.

\section{DISCUSSION}

In the present study we demonstrate that the additional assessment of ERCC1-transcripts enhanced overall CTC detection rate in ovarian cancer patients. It also defines an additional overlapping fraction of ERCC1expressing CTCs, which are potentially selected by platinum-based chemotherapy. We also describe that the assessment of CTC-derived ERCC1-transcripts alone was almost equivalently sufficient in order to detect ERCC1expressing prognostic relevant CTCs. We further showed
A

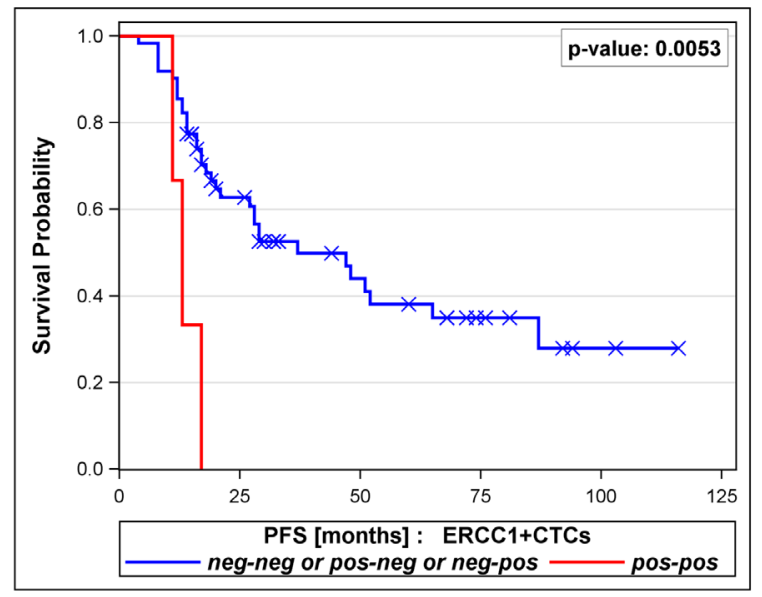

C

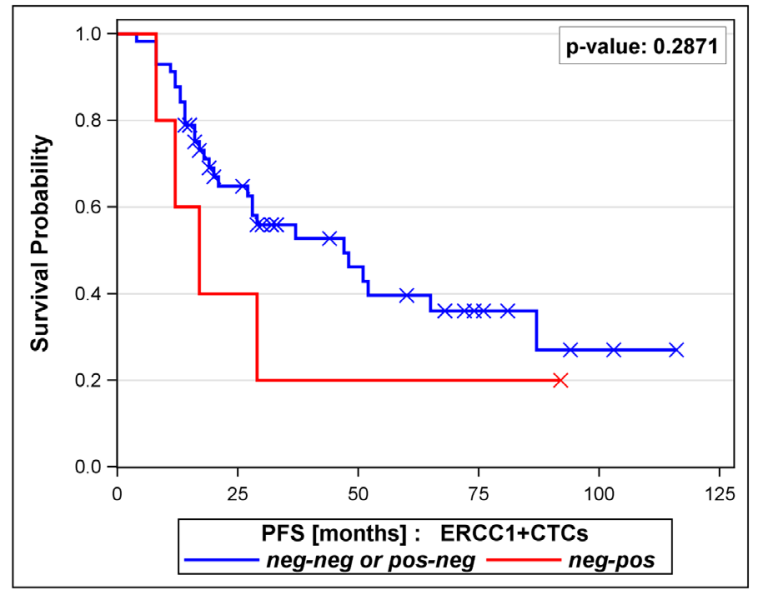

B

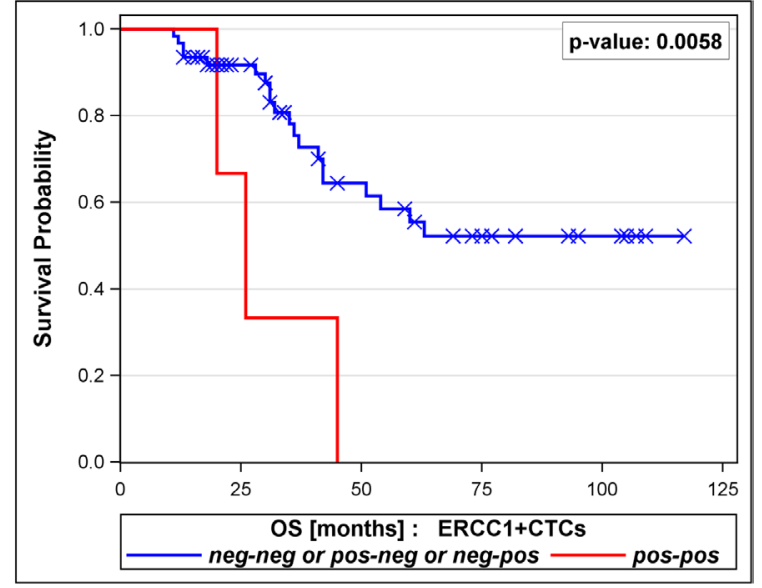

D

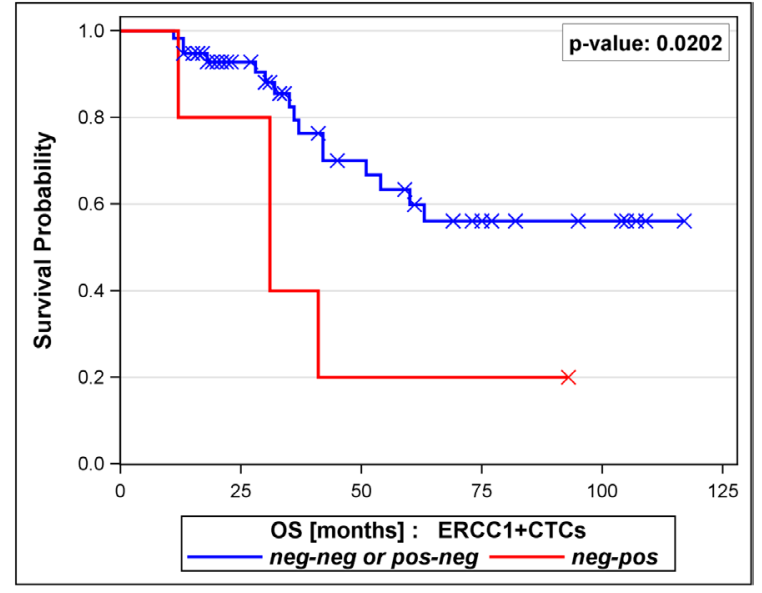

Figure 4: Prognostic relevance of persistent ERCC1 ${ }^{+}$CTCs. The Kaplan-Meier plots show A. progression-free survival and B. overall survival of patients with persistent positivity for $\mathrm{ERCC} 1{ }^{+} \mathrm{CTCs}$ in their blood (ERCC1 ${ }^{+} \mathrm{CTC}$ pos-pos, bottom curves), in comparison to all other dynamic subgroups together (ERCC1 ${ }^{+} \mathrm{CTC}$ pos-neg / neg-pos / neg-neg, top curves). Moreover, Kaplan-Meier plots show C. progression-free survival and $\mathbf{D}$. overall survival of patients with newly acquired positivity for ERCC $1{ }^{+} \mathrm{CTCs}(\mathrm{ERCC} 1+\mathrm{CTC}$ neg-pos, bottom curves), in comparison to the dynamic subgroups ERCC1 $1^{+} \mathrm{CTC}$ pos-neg and ERCC1 $1^{+} \mathrm{CTC}$ neg-neg, together (top curves). 
that the presence of ERCC $1^{+} \mathrm{CTCs}$ after chemotherapy correlated with post-therapeutic outcome of ovarian cancer and, particularly, dynamics of $\mathrm{ERCC} 1^{+} \mathrm{CTCs}$ mirrored response to platinum-based chemotherapy.

We have already demonstrated that a) additional detection of ERCC1-transcripts extented clinical value of CTCs from a prognostic biomarker to an independent predictor of platinum-resistance at primary diagnosis of ovarian cancer and b) ERCC $1^{+} \mathrm{CTCs}$ may constitute a distinct subgroup of CTCs with a potentially platinumresistant phenotype [21]. We now confirmed that auxiliary assessment of ERCC1-transcripts considerably expanded the phenotypic spectrum of CTC-detection in pre- and post-therapeutic blood samples and that ERCC1 obviously marks a definable CTC-phenotype with overlap to the CTC-population, as detected by the AdnaTest Ovarian Cancer. Therefore, in a considerable number of patients, CTC-derived ERCC1-expression was accompanied by co-expression of at least one of the standard markers for CTC-detection (EpCAM or MUC-1 or CA-125). Given the experimental setting of the AdnaTest, we cannot distinguish whether this coexpression was derived from CTCs actually co-expressing these markers on the same cell, or from separate CTCpopulations which were concomitantly present in the "pool" of immunomagnetically enriched CTCs from a given blood sample. However, we also observed a minor subset of patients who were exclusively positive for ERCC1-transcripts. We suppose that these patients harbor epithelial-associated CTCs in their blood, which express EpCAM or MUC-1 antigens on their surface. These CTCs were captured by the AdnaTest selection procedure which targets EpCAM and MUC-1 surface epitopes. However EpCAM and MUC-1 transcripts
A

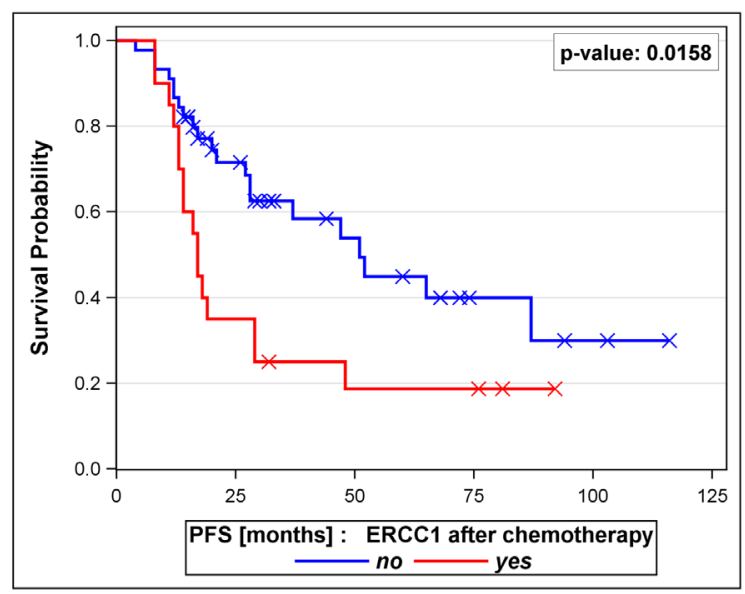

C

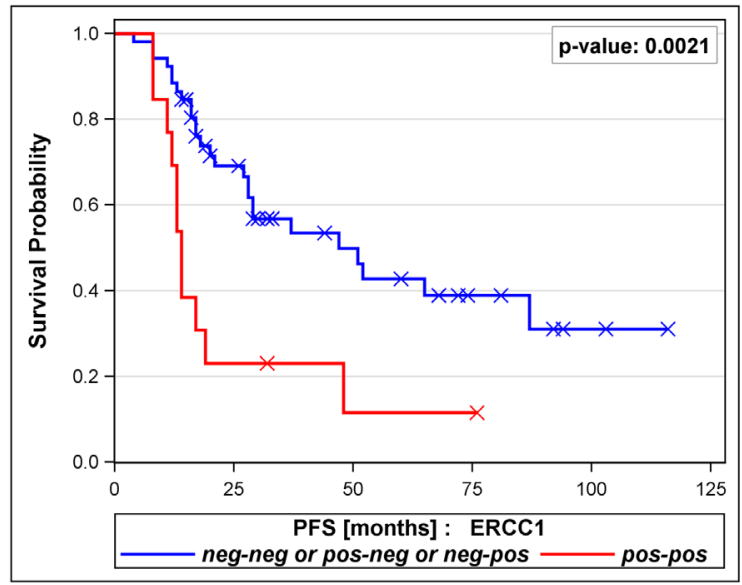

B

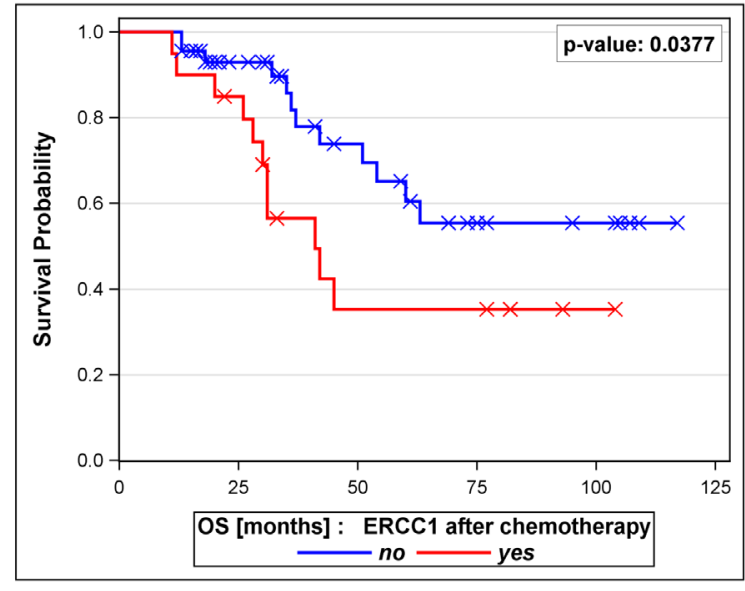

D

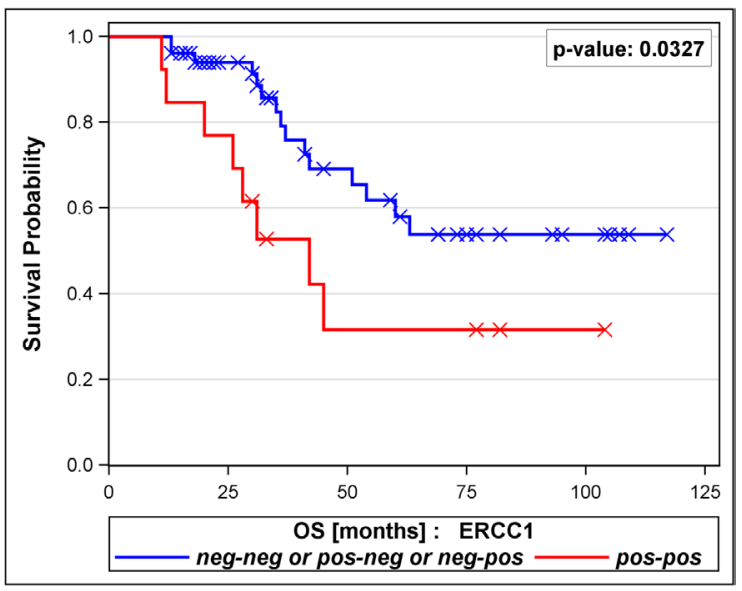

Figure 5: Prognostic relevance of ERCC1-transcripts alone. This analysis refers to the prognostic relevance of ERCC1-transcripts alone, irrespectively of the Adnatest transcript markers EpCAM, MUC-1 or CA-125. The Kaplan-Meier plots show A. progression-free survival and B. overall survival of patients with ERCC1-positivity after platinum-based chemotherapy (bottom curves) in comparison to patients with non-detectable ERCC1-transcripts (top curves). Moreover, Kaplan-Meier plots show C. progression-free survival and D. overall survival of patients with persistent positivity for ERCC1-transcripts (ERCC1 ${ }^{+}$pos-pos, bottom curves) in comparison to all other dynamic subgroups together (ERCC1 ${ }^{+}$pos-neg / neg-pos / neg-neg, top curves). 
seemed to be downregulated on the transcriptional level in these isolated CTCs. Discordances between protein and transcript expression profiles of a cell could be due to post-transcriptional modifications of messenger RNA or differences in the half-life time between messenger RNA and their corresponding proteins [22-24].

The broad heterogeneity of CTCs in the blood of cancer patients, including ovarian cancer, has already been indicated by several independent reports [25-27]. We may hypothesize that ERCC1-expressing CTCs play a dominant role during the course of the disease, which is corroborated by the fact that the rate of exclusively ERCC1-positive CTCs did not decrease after platinumbased chemotherapy. We have already shown that breast cancer patients with CTCs detected after neoadjuvant chemotherapy were associated with tumor stem cell characteristics and ERCC1-expression [28]. This may suggest a potential selection of this CTC-subset by chemotherapy. However, due to the fact that exclusively ERCC1-expressing CTCs rarely occurred in our patient cohort, we were not able to analyze prognostic significance of this particularly interesting CTC-subset in a statistically substantiated manner.

Independent of ERCC1 assessment, we previously confirmed the negative prognostic impact of CTCs before surgery and after chemotherapy, as detected by the AdnaTest Ovarian Cancer [21, 29]. Nevertheless, our recent and current results strongly support our perception that the auxiliary assessment of ERCC1transcripts provides complementary clinical information. In addition, the auxiliary assessment of ERCC1-transcripts after chemotherapy alone, as well as their expression dynamics in pre- and post-therapeutic blood samples, was almost equivalently sufficient as surrogate for a CTC-population. This might be useful for predicting posttherapeutic outcome and for monitoring platinum-based chemotherapy. Given the additional strong prognostic impact of the standard AdnaTest marker transcripts MUC1, EpCAM and CA-125 [21, 29] and considering that the statistical significance level slightly declined when only ERCC1-transcripts were assessed, a combined condition, which assumes ERCC1-positivity in addition to the detection of at least one of the AdnaTest markers (referred to as ERCC $1^{+} \mathrm{CTCs}$ in our study), appears to be most favorable in terms of a blood-based prognostic biomarker.

So far, any functional characteristics of ERCC1expressing CTCs in the blood of ovarian cancer patients are unknown. Since our study was performed exclusively from a "biomarker perspective", we can only assume that ERCC1-(over)expressing CTCs in the blood may be characterized by an enhanced, preexisting or newly acquired capacity to resolve DNA-platinum-adducts, consequently bypassing cisplatin-mediated cytotoxicity and possibly converting to a molecular phenotype of "on-target" platinum-resistance [8]. This assumption is further supported by a recent investigation which directly analyzed the presence of DNA-platinum adducts in single CTCs of advanced non-small cell lung cancer (NSCLC) patients. In this context, it was suggested that the kinetics of these adducts in pre- and post- therapeutic blood samples could be a potential biomarker for response prediction and dose individualization of platinum-based chemotherapy [30]. Consecutively, ERCC $1^{+} \mathrm{CTCs}$ may survive multiple cycles of chemotherapy and, in line with the fact that metastasis-initiating cells can be present among CTCs in the blood [31], persistent ERCC $1{ }^{+} \mathrm{CTCs}$ with a platinum-resistant phenotype could have the potential to initiate recurrence, resulting in poor clinical outcome. Taking into consideration that $\mathrm{ERCC} 1^{+} \mathrm{CTCs}$ are strong prognostic factors in the post-therapeutic situation, particularly in case of persistent positivity, our data may also indicate that platinum-resistant $\mathrm{ERCC} 1{ }^{+} \mathrm{CTC}$ s could be directly selected upon platinum-based chemotherapy. Further functional studies will be necessary in order to prove this hypothesis. Another interesting question for future studies will be, how CTC-derived ERCC1expression is related to EMT- and stem-like characteristics of CTCs. In this regard, we recently demonstrated that the negative prognostic impact of the presence and/ or persistence of disseminated tumor cells in the bone marrow of ovarian cancer patients after platinum based chemotherapy was associated with stem cell character [32].

Conclusively, due to the limited number of patients, our study is explorative and hypothesis generating. Nevertheless, ERCC1 marks a subpopulation of CTCs which might be useful for monitoring platinum-based chemotherapy and for assessing post-therapeutic outcome of ovarian cancer patients. We provide rationale to validate clinical utility of ERCC $1^{+} \mathrm{CTCs}$ among large multicenter clinical trials and to further elucidate their functional and tumor biological significance. Alternatively, patients with ERCC $1 *$ CTCs may profit from an early initiated and dose-intense maintenance therapy with e.g. Bevacizumab or PARP-inhibitors. Furthermore, this high risk patient group might be amenable to platinum-sensitizing therapies in the future, which are increasingly proposed in preclinical studies [33-35] and already ongoing clinical trials (NCT01164995). Using CTCs as liquid biopsy tool for individual therapy optimization, a multi-marker gene panel, compromising all CTC subgroups, will be useful to monitor patients during the course of the disease [36].

\section{PATIENTS AND METHODS}

\section{Patient characteristics}

The present study was conducted at the Department of Gynecology and Obstetrics at the University Hospital of Essen, Germany. A total of 65 patients diagnosed between 2006 and 2014 with histologically confirmed epithelial ovarian cancer were analyzed. Clinical characteristics 
of the patients are documented in Table 1. Informed written consent was obtained from all patients and the study was approved by the Local Ethics Committee (052870) and performed according to the declaration of Helsinki. Tumors were classified according to the WHO classification of tumors of the female genital tract. Grading was conducted using the grading system proposed by Silverberg [37] and tumor staging was classified according to the Fédération Internationale de Gynécology et d'Obstétrique [38]. The whole study population underwent primary radical surgery. Total abdominal hysterectomy, bilateral salpingo-oophorectomy, infragastric omentectomy, peritoneal stripping were performed. The most important aim of surgery was to achieve macroscopic complete tumor resection. Radical pelvic and para-aortic lymphadenectomy were only performed if macroscopic complete tumor resection was achieved intraperitoneally following actual guidelines. All patients received at least six cycles of carboplatinum AUC 5 and paclitaxel $175 \mathrm{mg} /$ $\mathrm{m}^{2}$ Tumors were clinically defined as platinum-resistant if they recurred within six months after the completion of platinum-based chemotherapy.

\section{Enrichment and molecular characterization of CTCs}

Peripheral blood $(2 \times 5 \mathrm{ml})$ from each patient was collected in EDTA tubes (Sarstedt \& Co.) and processed within $4 \mathrm{~h}$ for the enrichment of CTCs and subsequent expression analysis according to Adnatest Ovarian Cancer (QIAGEN, Hannover GmbH, Langenhagen, Germany). The test has been described in detail [21]. Briefly, CTCs were immunomagnetically selected using the AdnaTest Ovarian Cancer Select targeting epithelial cell adhesion molecule EpCAM (also known as GA7332), Mucin-1, cell surface associated (MUC-1) and cell surface associated Mucin-16 (also known as CA-125). Subsequently, RNA was isolated and gene expression analysis was performed by reverse-transcription (RT) and multiplex RT-PCR detecting EpCAM, MUC-1, and CA-125 (AdnaTest Ovarian Cancer Detect). ERCC1transcripts were investigated in a separate approach by singleplex RT-PCR. $\beta$-actin served as an internal control and PCR-products were quantified on the Agilent Bioanalyzer as follows: Blood samples of 20 healthy donors and healthy donor blood samples spiked with two or five cells IGROV1 were analyzed using the AdnaTest Ovarian Cancer Select/Detect for overexpression of EpCAM, MUC-1 and CA-125. The resulting PCR fragments were analyzed with the Agilent Bioanalyzer and the resulting data were checked for sensitivity and specificity to be $\geq 90 \%$ applying a $0.15 \mathrm{ng} / \mu \mathrm{l}$ fragment concentration as a cut of value for each of the markers. For the required specificity of $>90 \%$, as defined in the test performance criteria, the resulting cut off value was defined as $0.15 \mathrm{ng} / \mu \mathrm{l}$ fragment concentration. At a cut-off value of $0.15 \mathrm{ng} / \mu 1$ specificity is $95 \%$ and the corresponding recovery rate is $80 \%$ for two cells and $100 \%$ for five cells, respectively.

ERCC1-positivity was defined by an amplicon concentration $>0.2 \mathrm{ng} / \mu \mathrm{l}$. Sensitivity and specificity were evaluated in 20 healthy donors and 99 patients with primary ovarian cancer using ROC analysis. At a cut-off value of $0.17 \mathrm{ng} / \mu 1,95 \%$ specificity is reached and the corresponding clinical sensitivity is $46.5 \%$ (Supplementary Figure S2).

Amplicons with the following sizes were generated: EpCAM: 396bp; MUC-1: 293bp; CA-125: 432bp; ERCC1: 366bp; and $\beta$-actin: 114bp.

\section{Statistical analysis}

Survival curve plots and Hazard Ratio calculations were done using SAS (9.4). Survival intervals were screened from the time of CTC detection at first diagnosis to the time of clinical event (either death or first time of relapse) or last contact. Kaplan-Meier curves were assessed using the log-rank test to evaluate univariate significance of the binary grouping parameters. Fisher exact tests were performed to confirm significance. Survival curve plots and Hazard Ratio calculations were done using SAS (9.4).

\section{CONFLICTS OF INTEREST}

Sabine Kasimir-Bauer is a consultant for QIAGEN Hannover GmbH. Siegfried Hauch is an employee of QIAGEN Hannover GmbH. All other authors have no conflicts of interest.

\section{FUNDING}

The study was supported by the DFG (Deutsche Forschungsgemeinschaft), KA 1583/3-1.

\section{REFERENCES}

1. Goodman MT, Howe HL, Tung KH, Hotes J, Miller BA, Coughlin SS, Chen VW. Incidence of ovarian cancer by race and ethnicity in the United States, 1992-1997. Cancer. 2003; 97:2676-2685.

2. du Bois A, Quinn M, Thigpen T, Vermorken J, AvallLundqvist E, Bookman M, Bowtell D, Brady M, Casado A, Cervantes A, Eisenhauer E, Friedlaender M, Fujiwara $\mathrm{K}$, et al. 2004 consensus statements on the management of ovarian cancer: final document of the 3rd International Gynecologic Cancer Intergroup Ovarian Cancer Consensus Conference (GCIG OCCC 2004). Ann Oncol. 2005; 16:viii7-viii12.

3. du Bois A, Reuss A, Pujade-Lauraine E, Harter P, RayCoquard I, Pfisterer J. Role of surgical outcome as 
prognostic factor in advanced epithelial ovarian cancer: a combined exploratory analysis of 3 prospectively randomized phase 3 multicenter trials: by the Arbeitsgemeinschaft Gynaekologische Onkologie Studiengruppe Ovarialkarzinom (AGO-OVAR) and the Groupe d'Investigateurs Nationaux Pour les Etudes des Cancers de l'Ovaire (GINECO). Cancer. 2009; 115:1234-1244.

4. Wimberger P, Lehmann N, Kimmig R, Burges A, Meier W, Du Bois A; Arbeitsgemeinschaft Gynaekologische Onkologie Ovarian Cancer Study G. Prognostic factors for complete debulking in advanced ovarian cancer and its impact on survival. An exploratory analysis of a prospectively randomized phase III study of the Arbeitsgemeinschaft Gynaekologische Onkologie Ovarian Cancer Study Group (AGO-OVAR). Gynecol Oncol. 2007; 106:69-74.

5. Wimberger $P$, Wehling $M$, Lehmann $N$, Kimmig $R$, Schmalfeldt B, Burges A, Harter P, Pfisterer J, du Bois A. Influence of residual tumor on outcome in ovarian cancer patients with FIGO stage IV disease: an exploratory analysis of the AGO-OVAR (Arbeitsgemeinschaft Gynaekologische Onkologie Ovarian Cancer Study Group). Ann Surg Oncol. 2010; 17:1642-1648.

6. Martin LP, Schilder RJ. Management of recurrent ovarian carcinoma: current status and future directions. Semin Oncol. 2009; 36:112-125.

7. Bookman MA. Extending the platinum-free interval in recurrent ovarian cancer: the role of topotecan in secondline chemotherapy. Oncologist. 1999; 4:87-94.

8. Galluzzi L, Senovilla L, Vitale I, Michels J, Martins I, Kepp O, Castedo M, Kroemer G. Molecular mechanisms of cisplatin resistance. Oncogene. 2012; 31:1869-1883.

9. Britten RA, Liu D, Tessier A, Hutchison MJ, Murray D. ERCC1 expression as a molecular marker of cisplatin resistance in human cervical tumor cells. Int J Cancer. 2000; 89:453-457.

10. Grimminger PP, Shi M, Barrett C, Lebwohl D, Danenberg KD, Brabender J, Vigen CL, Danenberg PV, Winder T, Lenz HJ. TS and ERCC-1 mRNA expressions and clinical outcome in patients with metastatic colon cancer in CONFIRM-1 and -2 clinical trials. Pharmacogenomics J. 2012; 12:404-411.

11. Liu YP, Ling Y, Qi QF, Zhang YP, Zhang CS, Zhu CT, Wang MH, Pan YD. The effects of ERCC1 expression levels on the chemosensitivity of gastric cancer cells to platinum agents and survival in gastric cancer patients treated with oxaliplatin-based adjuvant chemotherapy. Oncol Lett. 2013; 5:935-942.

12. Olaussen KA, Dunant A, Fouret P, Brambilla E, Andre F, Haddad V, Taranchon E, Filipits M, Pirker R, Popper HH, Stahel R, Sabatier L, Pignon JP, et al. DNA repair by ERCC1 in non-small-cell lung cancer and cisplatin-based adjuvant chemotherapy. N Engl J Med. 2006; 355:983-991.
13. Arora S, Kothandapani A, Tillison K, Kalman-Maltese V, Patrick SM. Downregulation of XPF-ERCC1 enhances cisplatin efficacy in cancer cells. DNA Repair (Amst). 2010; 9:745-753.

14. Milovic-Kovacevic M, Srdic-Rajic T, Radulovic S, Bjelogrlic S, Gavrilovic D. Expression of ERCC1 protein in biopsy specimen predicts survival in advanced ovarian cancer patients treated with platinum-based chemotherapy. J BUON. 2011; 16:708-714.

15. Scheil-Bertram S, Tylus-Schaaf $\mathrm{P}$, du Bois A, Harter P, Oppitz M, Ewald-Riegler N, Fisseler-Eckhoff A. Excision repair cross-complementation group 1 protein overexpression as a predictor of poor survival for highgrade serous ovarian adenocarcinoma. Gynecol Oncol. 2010; 119:325-331.

16. Xie C, Yin RT, Li YL, Kang DY, Xu L, Yang KX. [The protein expression of ERCC1 and survivin in epithelial ovarian carcinoma and their clinical significance] [Article in Chinese]. Sichuan Da Xue Xue Bao Yi Xue Ban. 2011; 42:86-89.

17. Vandenput I, Capoen A, Coenegrachts L, Verbist G, Moerman P, Vergote I, Amant F. Expression of ERCC1, p53, and class III beta-tubulin do not reveal chemoresistance in endometrial cancer: results from an immunohistochemical study. Int J Gynecol Cancer. 2011; 21:1071-1077.

18. Bosmuller H, Haitchi-Petnehazy S, Webersinke G, Marschon R, Roithmeier F, Stummvoll W, Fehm T, KlierRichter M, Bonzheim I, Staebler A, Fend F. Intratumoral lymphocyte density in serous ovarian carcinoma is superior to ERCC1 expression for predicting response to platinumbased therapy. Virchows Arch. 2011; 459:183-191.

19. Hubner RA, Riley RD, Billingham LJ, Popat S. Excision repair cross-complementation group 1 (ERCC1) status and lung cancer outcomes: a meta-analysis of published studies and recommendations. PLoS One. 2011; 6:e25164.

20. Friboulet L, Olaussen KA, Pignon JP, Shepherd FA, Tsao MS, Graziano S, Kratzke R, Douillard JY, Seymour L, Pirker R, Filipits M, Andre F, Solary E, et al. ERCC1 isoform expression and DNA repair in non-small-cell lung cancer. New Engl J Med. 2013; 368:1101-1110.

21. Kuhlmann JD, Wimberger P, Bankfalvi A, Keller T, Scholer S, Aktas B, Buderath P, Hauch S, Otterbach F, Kimmig R, Kasimir-Bauer S. ERCC1-positive circulating tumor cells in the blood of ovarian cancer patients as a predictive biomarker for platinum resistance. Clin Chem. 2014; 60:1282-1289.

22. Pascal LE, True LD, Campbell DS, Deutsch EW, Risk M, Coleman IM, Eichner LJ, Nelson PS, Liu AY. Correlation of mRNA and protein levels: cell type-specific gene expression of cluster designation antigens in the prostate. BMC Genomics. 2008; 9:246.

23. Greenbaum D, Colangelo C, Williams K, Gerstein M. Comparing protein abundance and mRNA expression levels on a genomic scale. Genome Biol. 2003; 4:117. 
24. Nie L, Wu G, Zhang W. Correlation of mRNA expression and protein abundance affected by multiple sequence features related to translational efficiency in Desulfovibrio vulgaris: a quantitative analysis. Genetics. 2006; 174:2229-2243

25. Blassl C, Kuhlmann JD, Webers A, Wimberger P, Fehm T, Neubauer H. Gene expression profiling of single circulating tumor cells in ovarian cancer - Establishment of a multimarker gene panel. Mol Oncol. 2016. 10:1030-42.

26. De Luca F, Rotunno G, Salvianti F, Galardi F, Pestrin M, Gabellini S, Simi L, Mancini I, Vannucchi AM, Pazzagli M, Di Leo A, Pinzani P. Mutational analysis of single circulating tumor cells by next generation sequencing in metastatic breast cancer. Oncotarget. 2016. 3;7:26107-19. doi: 10.18632/oncotarget.8431.

27. Brouwer A, De Laere B, Peeters D, Peeters M, Salgado R, Dirix L, Van Laere S. Evaluation and consequences of heterogeneity in the circulating tumor cell compartment. Oncotarget. 2016; 7:48625-48643. doi: 10.18632/oncotarget.8015.

28. Kasimir-Bauer S, Bittner AK, Konig L, Reiter K, Keller T, Kimmig R, Hoffmann O. Does primary neoadjuvant systemic therapy eradicate minimal residual disease? Analysis of disseminated and circulating tumor cells before and after therapy. Breast Cancer Res. 2016; 18:20.

29. Aktas B, Kasimir-Bauer S, Heubner M, Kimmig R, Wimberger P. Molecular profiling and prognostic relevance of circulating tumor cells in the blood of ovarian cancer patients at primary diagnosis and after platinum-based chemotherapy. Int J Gynecol Cancer. 2011; 21:822-830.

30. Nel I, Gauler TC, Eberhardt WE, Nickel AC, Schuler M, Thomale J, Hoffmann AC. Formation and repair kinetics of Pt-(GpG) DNA adducts in extracted circulating tumour cells and response to platinum treatment. Br J Cancer. 2013; 109:1223-1229.

31. Baccelli I, Schneeweiss A, Riethdorf S, Stenzinger A, Schillert A, Vogel V, Klein C, Saini M, Bauerle T,
Wallwiener M, Holland-Letz T, Hofner T, Sprick M, et al. Identification of a population of blood circulating tumor cells from breast cancer patients that initiates metastasis in a xenograft assay. Nat Biotechnol. 2013; 31:539-544.

32. Chebouti I, Blassl C, Wimberger P, Neubauer H, Fehm T, Kimmig R, Kasimir-Bauer S. Analysis of disseminated tumor cells before and after platinum based chemotherapy in primary ovarian cancer. Do stem cell like cells predict prognosis? Oncotarget. 2016; 7:26454-26464. doi: 10.18632/oncotarget.8524.

33. Jazaeri AA, Shibata E, Park J, Bryant JL, Conaway MR, Modesitt SC, Smith PG, Milhollen MA, Berger AJ, Dutta A. Overcoming platinum resistance in preclinical models of ovarian cancer using the neddylation inhibitor MLN4924. Mol Cancer Ther. 2013; 12:1958-1967.

34. Peng DJ, Wang J, Zhou JY, Wu GS. Role of the Akt/mTOR survival pathway in cisplatin resistance in ovarian cancer cells. Biochem Biophys Res Commun. 2010; 394:600-605.

35. Fukushima H, Abe T, Sakamoto K, Tsujimoto H, Mizuarai S, Oie S. 3'-ethynylcytidine, an RNA polymerase inhibitor, combined with cisplatin exhibits a potent synergistic growth-inhibitory effect via Vaults dysfunction. BMC Cancer. 2014; 14:562.

36. Bredemeier M, Edimiris P, Tewes M, Mach P, Aktas B, Hauch S, Wagner J, Kimmig R, Kasimir-Bauer S. A. Establishment of a multimarker qPCR panel for the molecular characterization of circulating tumor cells in blood samples of metastatic breast cancer patients during the course of palliative treatment. Oncotarget. 2016; 7:41677-41690. doi: 10.18632/oncotarget.9528.

37. Silverberg SG. Histopathologic grading of ovarian carcinoma: a review and proposal. Int J Gynecol Pathol. 2000; 19:7-15.

38. Current FIGO staging for cancer of the vagina, fallopian tube, ovary, and gestational trophoblastic neoplasia. Int $\mathrm{J}$ Gynaecol Obstet. 2009; 105:3-4. 